\title{
Switching between the Forest and the Trees: Brain Systems Involved in Local/Global Changed-Level Judgments
}

\author{
David T. Wilkinson, Peter W. Halligan,*† J ohn C. Marshall, ‡ Christian Büchel,§ and Raymond J . Dolan§, \\ Department of Experimental Psychology, Oxford University, Oxford OX1 3UD, United Kingdom; *Rivermead Rehabilitation Centre, \\ Oxford, OX1 4XD, United Kingdom; †School of Psychology, Cardiff University, Cardiff CF 10 3YG, United Kingdom; ¥Neuropsychol ogy \\ Unit, University Department of Clinical Neurology, The Raddiffe Infirmary, Woodstock Road, Oxford OX2 6HE, United Kingdom; \\ §Wel Icome Department of Cognitive Neurology, Institute of Neurology, London WC1N 3BG, United Kingdom; and \\ "Royal Free Hospital School of Medicine, Roland Hill Street, London NW3 2DF, United Kingdom
}

Received April 17, 2000; published online November 15, 2000

\begin{abstract}
Visual targets can be coded, in relative terms, at either the local or the global level of stimuli. Previous studies have indicated that targets are identified more slowly when they appear at a new hierarchical level, compared to when they reappear at the same level as in the previous trial. In the present study, we used measures of reaction time and event-related fMRI to investigate factors affecting this switch cost. In particular, we examined the effects of the number of repeated-level trials preceding a switch and whether the cue to switch was either externally or internally mediated. At the behavioral level we found that (1) the time taken to identify a target on a changed-level trial is longer following four repeated-level trials compared to two repeated-level trials, but that runs of six do not produce additional costs over four, and (2) targets can be identified faster following externally cued switches compared to internally mediated switches. We then show that these behavioral effects are associated with distinct patterns of neural activation. Switches performed after two repeated-level trials preferentially activated the precuneus, while those performed after both four and six activated bilateral inferior parietal cortex and motor hand area. Relative to external switches, internal switches activated the putamen, while both kinds of switch conjointly activated the large-scale network proposed to underlie internal/external switches in nonhierarchical tasks. Our data further clarify the mechanisms mediating hierarchical selection. 2001 Academic Press
\end{abstract}

\section{INTRODUCTION}

The spatial relations of objects have been described in categorical terms (i.e., above- below or left-right) or in terms of parts and wholes (i.e., a hub is part of a wheel, which in turn is part of a car). ${ }^{1}$ There is now considerable evidence that parts and wholes are re spectively coded by separate psychol ogical mechanisms (e.g., Navon, 1977; Palmer, 1977) which are associated with discrete cerebral loci (Delis et al., 1986; Robertson et al., 1988). One aspect of part/whole, or local/global, processing concerns switching between hierarchical levels. It has been observed in previous studies that a time cost is associated with identifying targets which appear at a changed hierarchical level, compared to when they reappear at the same hierarchical level as on the previous trial (N. Kim et al., 1999; Lamb and Yund, 1996; Lamb et al., 1999; Robertson et al., 1988, 1993; Ward, 1982). Clues as to the cerebral loci underlying this switch have been observed in patients with lesions to the left inferior parietal lobe, who fail to show this switch cost and seem insensitive to the hierarchical level at which the target last appeared (Robertson et al., 1988). In the present study, we further investigated switch costs in local/global processing. A switch can occur within the context of previous switches, while the actual cue to switch can be either external or internal in nature. We examine the interaction of these factors with the magnitude of switch cost. At the level of neural activation, little is known about how these contextual influences interact with the structures that mediate judgments on changed-level trials.

One question that arises is whether the magnitude of cost associated with a changed-level trial is affected by the number of times a target has been consecutively identified at the same level prior to a switch. In a post hoc analysis, Robertson (1996) examined whether performance was affected by the second-to-last trial pre-

\footnotetext{
${ }^{1}$ This verbal distinction is not intended to reflect a true dichotomy, since visual input can be decomposed into multiple hierarchical levels. Accordingly, the term "local/global" is used in a descriptive sense to refer to the relative distinction between hierarchical levels.
} 
ceding a switch. When the target appeared at a level for only one trial before switching levels, reaction times (RTs) were shorter than when the target had remained at that level for two trials. This indicated that the number of repeated-level trials preceding a switch may modulate switch performance. In her Attentional Print model, Robertson (1996, 1999) proposes that information is carried over from the last trial by the relative priming of spatial frequency channels. Whichever spatial frequency channel last resolved the target receives a greater attentional weighting in resolving the next target, leading to benefits for repeated-level over changed-level trials. I mportantly, the model predicts that the greater the number of repeated-level trials preceding a changed-level trial, the greater the switch cost.

At the anatomical level, the left inferior parietal lobe has been associated with the carrying over of target level information from the last trial, as damage to this area eliminates level-specific priming (Robertson et al., 1988). Nonetheless, the failure of the study to test patients with homologous right inferior parietal damage leaves open the possibility that the process is instantiated bilaterally. For example, there is considerable evidence that the right parietal lobe is dominant in spatial processing; more severe deficits in covert disengagement of attention can occur following rightrather than left-sided lesions (Posner et al., 1984), and the incidence of spatial neglect is more common following right-sided lesions (Halligan and Marshall, 1994). By using event-related $\mathrm{FMRI}$ it is possible to test both for any right hemisphere involvement in the carryover of level-specific information and whether activity in the left inferior parietal lobe is modulated by the number of repeated-level trials preceding a switch.

One other psychological factor that may affect the magnitude of this switch cost is whether the cue to switch is either internally or externally generated. The Navon figures used in local/global experiments often contain external cues that facilitate judgments at one particular level. This precedence of one level over the other does not appear to be intentionally mediated, but is rather the result of stimulus factors such as the sparsity of local elements (Martin, 1979), retinal Iocus (Lamb and Robertson, 1988), and exposure duration (Boles and Karner, 1996). The observation that RTs are shorter for targets that appear at the level of precedence (Martin, 1979) is perhaps analogous to the external shifts of attention described in spatial cuing experiments (Posner et al., 1980). In these studies, attention is automatically, or reflexively, pulled to a salient target, such as a peripheral, flashing, bright light. This contrasts with a slower, nonreflexive internal switch in which a centrally appearing cue (i.e., an arrow) indicates the direction in which the individual must look to find the subsequent target. In a similar way, the operation of precedence might be considered as externally cuing processing to a specific hierarchical level. Thus, in a global precedence stimulus, the identification of global targets would rely only on a reflexive cue, while the identification of local targets would take longer as it required a second, self-initiated cue which must overcome the effects of precedence. Likewise, in a local precedence stimulus, the identification of local targets would be reliant on a reflexive cue, while the identification of global targets would require an internal cue.

The neural basis of external and internal switching has not been examined in local/global stimuli. They have, however, been investigated in spatial cuing tasks in which subjects attend to features briefly presented in the visual periphery. Typically, both kinds of switches activate a large-scale network composed of the frontal eye fields and posterior parietal, anterior cingulate, and premotor cortices (Y. H. Kim et al ., 1999; Nobre et al., 1997; Rosen et al., 1999). In these studies, relative to external switches, internal switches preferentially activated a region in the left posterior parietal cortex, globus pallidus, right dorsolateral prefrontal cortex, and cerebellum while relative to internal switches, external switches preferentially activated ventrothalamic nuclei. The external/internal switches in these studies were between simple visual stimuli (i.e., single features or basic form conjunctions) that occupied discrete, lateralized locations. It therefore remains unclear whether the pattern of activation obtained for these kinds of switches generalizes to switches between the local and the global levels of centrally presented, hierarchical stimuli.

Although numerous studies (e.g., Fink et al., 1998; Heinze et al ., 1998) have investigated those brain areas allocating attention to either the global or the local level of stimuli, there remains only one neuroimaging study that has examined local/gl obal switching (F ink et al., 1997, experiment 2). The task involved reporting at which level (local or global) a predesignated target had appeared, with target level alternating 1 to 34 times a minute. This contrasted with a directed attention task (Fink et al., 1997, experiment 1) in which subjects were required to name the letter appearing at the same level throughout. Changes in regional cerebral blood flow correlated with an increasing number of switches in left supplementary and medial parietal cortices, while sustained attention to local and global levels activated left and right temporoparietal regions, respectively. Although indicating that local/global switching generates a pattern of activation separate from that of sustained attention, the use of a block design rendered the experiment insensitive to the potential effects of levelspecific priming and external/internal cues on switch performance.

In the present study, subjects identified, by means of a button press, which of four targets $(A, E, O, U)$ had appeared in a hierarchical, incongruent Navon stimulus. We manipulated three factors: the number of re 


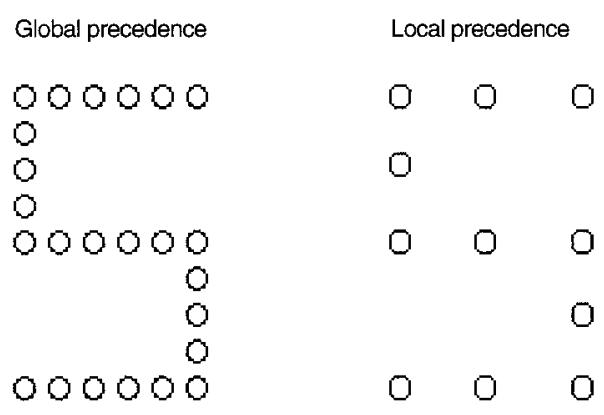

FIG. 1. Example stimuli showing a target " $O$ " at the local level under (i) global precedence and (ii) local precedence in a Navon incongruent figure.

peated-level trials preceding a switch ( 2 vs 4 vs 6 ), the level at which precedence occurred (local vs global), and the direction of switch (local to global vs global to local). At the behavioral level, we predicted that switch costs would be greater both as the number of repeatedlevel trials increased and in the internal condition compared to the external condition (as determined by the interaction between level precedence and switch direction). At the level of neural activation, we predicted that, in addition to left inferior parietal cortex, the right inferior parietal cortex would show level-specific priming effects. We also tested both whether external and internal local/global switches were conjointly mediated by the large-scale network underlying lateralized, nonhierarchical shifts of attention (N. Kim et al., 1999) and if internal and external cues produced the reported prefrontal and subcortical dissociations (Nobre et al., 1997; Rosen et al., 1997).

\section{METHOD}

\section{Subjects}

Six male and six female normal-sighted subjects were tested (mean age 30), all of whom were strongly right handed, yielding a group handedness mean of 23 of a maximum of 24, as tested by the Briggs and Nebes (1975) modified version of the Annett's Handedness Inventory.

\section{Stimuli}

Incongruent Navon figures were presented (see Fig. 1). Global letters subtended $\sim 9^{\circ} \times \sim 6^{\circ}$ visual angle and local letters subtended $\sim 0.8^{\circ} \times \sim 0.6^{\circ}$ visual angle at a viewing distance of approximately $30 \mathrm{~cm}$. Stimuli (black on a white background) appeared quasi-randomly in the middle of one of four quadrants located around the middle of the display. The center of each quadrant was positioned on one of four corners of an imaginary, centrally located square, which occupied $4^{\circ}$ $\times 4^{\circ}$ visual angle. Targets appeared at either the gl obal or the local level of stimuli. Global target stimuli con- sisted of As, Ah, Ep, Et, Ot, Os, Up, and Uh and local target stimuli of $\mathrm{Sa}, \mathrm{Ha}, \mathrm{Pe}, \mathrm{Te}, \mathrm{To}, \mathrm{So}, \mathrm{Pu}$, and $\mathrm{Hu}$ (in which uppercase and lowercase letters represent the global and local stimulus letters, respectively). There were two variants of each stimulus, one with global precedence and the other with local precedence (see Fig. 1). Each of these stimuli appeared with equal probability across the experiment and were presented in a pseudo-random sequence that prevented targets which utilized the same response button ( $A$ and $E$ or $O$ and $U$ ) from appearing in more than three consecutive trials. Although the target was always a vowel and the distracter a consonant, there is no evidence from the visual modality that this distinction could have been used as an external cue.

To examine the effects of external cues on switching, stimuli were constructed with strong precedence. Following Martin (1979) the local elements in global precedence stimuli were positioned very closely together, and care was taken to ensure that the global identity constituted a good exemplar of the target letter. In contrast, the local targets in local precedence stimuli were spaced wider apart. Precedence was then verified using two procedures. I nitially, printed versions of the hierarchical, incongruent stimuli were presented, each for $1 \mathrm{~s}$, to six subjects who were asked to say which letter they saw first. Stimuli were continually modified and presented to new groups of subjects until their responses accorded with the desired response on $90 \%$ of trials. A separate group of six subjects then performed a more formal, computerized task in which one of four possible targets had to be identified. Both the stimuli and the procedure were the same as that reported for the main experiment, with the exception that the ordering of global and local precedence stimuli was randomized, rather than being divided into separate runs (since here we were not concerned with the effects of the number of repeated-level trials preceding a judgment). Consistent with the results of the noncomputerized test, global targets were identified faster in the global precedence condition compared to the local precedence condition ( $t=3.95(5), P<0.01$ ) and local targets were identified faster in the local precedence condition than the global precedence condition (t $=4.77$ (5), $\mathrm{P}<0.01$ ). These data confirmed that the effects of precedence on RT were reliable.

\section{Procedure}

For each stimulus presentation, subjects were instructed to indicate (both as quickly and as accurately as possible) the presence of one of four targets ( $A, E, O$, $U$ ) by pressing the right button of a response box for $A$ or $\mathrm{E}$ and the left button for $\mathrm{O}$ or $\mathrm{U}$. Thirty-two practice trials were conducted outside of the scanner, in which each stimulus was presented, and the mapping of target to responding finger could be rehearsed. Further 


\begin{tabular}{|c|c|c|c|c|c|c|c|c|c|c|c|c|c|c|c|}
\hline \multirow{4}{*}{$\begin{array}{l}\text { Stimulus } \\
\text { Trial type } \\
\text { Switch type }\end{array}$} & \multicolumn{3}{|c|}{ Run I } & \multicolumn{2}{|c|}{ Run 2} & \multicolumn{6}{|c|}{ Run 3} & \multicolumn{4}{|c|}{ Run 4} \\
\hline & $\mathrm{Ah}$ & Os & $\mathrm{Ha}$ & $\mathrm{Pu}$ & $\mathrm{Ot}$ & $\mathrm{Et}$ & Up & Uh & Ep & As & To & $\mathrm{Pe}$ & So & $\mathrm{Pu}$ & Et \\
\hline & & ns & 2 & ns & 2 & ns & $\mathrm{ns}$ & ns & ns & $\mathrm{ns}$ & 6 & ns & $\mathrm{ns}$ & ns & 4 \\
\hline & & & in & & ex & & & & & & ex & & & & in \\
\hline & & & $\mathrm{g} / 1$ & & $1 / g$ & & & & & & $\mathrm{~g} / 1$ & & & & $1 / g$ \\
\hline$\underline{\text { Target level }}$ & $\mathrm{g}$ & $\mathrm{g}$ & 1 & 1 & g & $\mathrm{g}$ & $\mathrm{g}$ & $g$ & $\mathrm{~g}$ & $\mathrm{~g}$ & 1 & 1 & 1 & 1 & $\mathrm{~g}$ \\
\hline Prec' level & g & g & $\mathrm{g}$ & 1 & g & $\mathrm{g}$ & g & $\mathrm{g}$ & $\mathrm{g}$ & g & 1 & l & 1 & 1 & 1 \\
\hline
\end{tabular}

FIG. 2. Graphical illustration of a sequence of four experimental trials. The first two runs illustrate a switch after two repeated-level trials, the third a switch after six repeated-level trials, and the fourth a switch after four repeated-level trials. Note that Switch type is determined by the interaction of target level and precedence level. For each stimulus name, the uppercase letter refers to the global identity of the stimulus and the lowercase refers to its local identity. Abbreviations: ns, no switch; 2, switch after two repeated-level trials; 4 , switch after four repeated-level trials; 6, switch after six repeated-level trials; in, internal switch; ex, external switch; g/l, global to local switch; I/g, local to global switch; g, global; I, local; Prec', precedence level.

practice was provided on request. In the experiment proper, the ordering of targets was again randomized, and responding hand and fingers were counterbalanced across subjects. Responses on switch trials did not always coincide with a change in response button, and similarly, responses on repeated-level trials did not always coincide with repeated pressing of the same response button. Stimulus exposure duration was 150 ms. Within each run of trials (a run being defined as either 2, 4, or 6 repeated-level trials plus a switch), stimuli appeared every $2.5 \mathrm{~s}$, with white pre and postexposure fields. Different runs were first spaced an average of $10.8 \mathrm{~s}$ apart and then randomly jittered by \pm 0.5 TR (1.6 s) to fully sample the evoked hemodynamic responses. All switch types were distributed pseudorandomly across two 13-min experimental sessions with the constraint that the same switch trial never occurred more than twice in succession. The two sessions were divided by a short break.

\section{Experimental Manipulations}

Although target identity changed randomly, the hierarchical level at which these appeared was strictly controlled. Stimuli were presented in runs that comprised one, three, or five repeated-level trials, plus a changed-level trial. It can be seen from Fig. 2 that it was logical to also consider the last trial of the previous run a repeated-level trial. Thus, in effect, switches were preceded by two, four, or six repeated-level trials. The two and four repeated-level trial conditions occurred 33 times, and the six repeated-level trials occurred 34 times. Within any run of repeated-level trials, stimulus precedence and target location remained at the same hierarchical level.

Half of these 100 switches were externally cued and half were internally cued. In externally cued switches, target level and precedence level always corresponded. These were induced by changing both the level of stimulus precedence and the target from the previous repeated-level trial. For example, in a local-to-global ex- ternal switch, both precedence and target level changed to global, having both been set at the local level for the preceding repeated-level trials. In this way, targets could be identified by following the cues provided by stimulus precedence. In contrast, internally cued switches were induced by changing the target level but not the precedence level. Here, target identity would be reliant on an internally mediated switch which had to overcome the effects of precedence. For example, in a local-to-global internal switch, the target level changed from local on the previous trial to global on the present trial, but the precedence level remained at the local level. This manipulation also enabled us to examine the effects of switch direction. This could either be from the local level to the global level or from the global level to the local level. Switches in either direction were performed 50 times, and in consequence, there were 25 switches for each of the switch direction-switch cue pairings (internal local to global, internal global to local, external local to global, external global to local).

\section{Image Acquisition}

A 2-T Magnetom Vision system was used to acquire both 3D MP-RAGE sequence T1 anatomical images ( $1 \times 1 \times 1.5$-mm voxels) and T2*-weighted EPI images (3-mm in-plane resolution with a slice thickness of 2 $\mathrm{mm}$ and a gap of $1 \mathrm{~mm}$, yiel ding a resolution of $3 \times 3 \times$ $3 \mathrm{~mm}$ ). Each EPI image comprised 32 1.8-mm axial slices and employed a TE of $40 \mathrm{~s}$. Five hundred fifty volume images were acquired with a TR of $3.2 \mathrm{~s} / \mathrm{vol}$ ume. Each subject's scans were realigned to the first volume and resliced using sync interpolation, adjusting for residual motion-related signal changes. A mean image was then coregistered with a structural T1 volume and then spatially normalized to a standard template. The data were smoothed using a 10-mm (full width at half maximum) isotropic Gaussian kernel. 


\section{Data Analysis}

Data were analyzed using statistical parametric mapping (SPM 99) employing a random effects model. We specified 12 effects of interest: ${ }^{2}(1)$ external localto-global switch after two repeated-level trials, (2) external local-to-global switch after four repeated-level trials, (3) external local-to-global switch after six repeated-level trials, (4) external global-to-local switch after two repeated-level trials, (5) external global-tolocal switch after four repeated-level trials, (6) external global-to-local switch after six repeated-level trials, (7) internal local-to-global switch after two repeated-level trials, (8) internal local-to-global switch after four repeated-level trials, (9) internal local-to-global switch after six repeated-level trials, (10) internal global-tolocal switch after two repeated-level trials, (11) internal global-to-local switch after four repeated-level trials, (12) internal global-to-local switch after six repeated-level trials. These effects were modeled by convolving a $\delta$ function with the hemodynamic response function, and its temporal derivative, to create regressors of interest. The random-effects analysis involved three steps. Session-specific parameter estimates pertaining to the hemodynamic response function for each effect of interest were calculated for each voxel. Second, an appropriate contrast of parameter estimates across sessions was calculated in a voxelwise manner to produce, for each subject, one contrast for each experimental condition. Third, for each comparison of interest, the appropriate contrast images from each individual were entered into a one-sample t test performed across all 12 subjects. Unless otherwise specified, all reported statistical effects were thresholded at a significance level of $\mathrm{P}<0.05$, corrected for multiple comparisons.

\section{RESULTS}

\section{Behavioral Responses}

Mean correct RTs and arcsine transformed errors were computed for each subject. RTs that were more than 2.5 standard deviations from the mean were discarded (for each subject these accounted for no more than two responses per condition). To examine the effects of the number of repeated-level trials on switch performance, one-way repeated-measures ANOVAs (Number of Repeated-Level Trials: two vs four vs six) were performed on the RT and error data. A separate 2 (Switch Direction: local to global vs global to local) $\times 2$ (Switch Cue: internal vs external) repeated-measures

\footnotetext{
${ }^{2}$ Given the experimental rationale, these individual effects were never separately contrasted, as we were interested only in the main effects of Number of Repeated-Level Trials and Switch Cue and the two-way interactions between Switch Cue (External vs I nternal) and Switch Direction (Local to Global vs Global to Local).
}
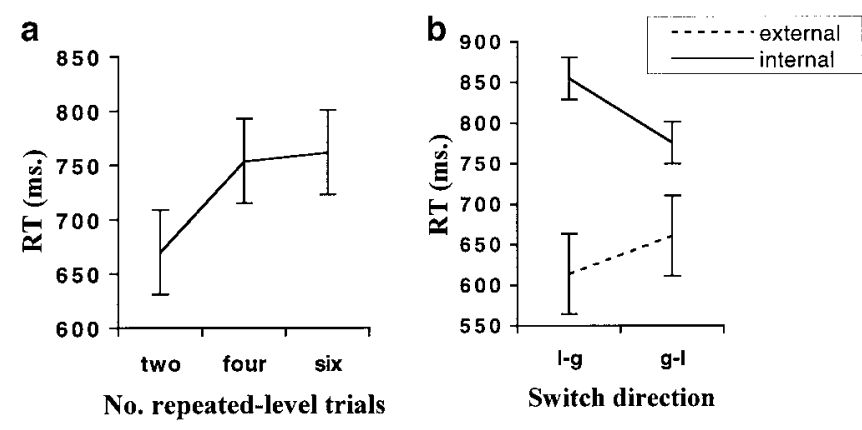

FIG. 3. Mean RT (with standard error bars) for changed-level trials showing performance as a function of (a) the number of preceding repeated-level trials and (b) switch cue and switch direction (I-g, local-to-global switch; g-l, global-to-local switch).

ANOVA was performed to examine the effects of switch direction, switch cue, and any possible interaction effects. All post hoc analyses were conducted using Newman-Keuls pairwise comparisons. We were unable to perform a single analysis across switch direction, switch cue, and number of repeated-level trials because there would have been too few data points $(\sim 7)$ per cell to conduct a meaningful analysis. ${ }^{3}$

\section{Repeated-Level Trials}

The RT main effect of Number of Repeated-Level Trials was significant $(F(2,22)=12.6$, Mse $=2461, \mathrm{P}<$ 0.01 ). Changed-level trials were performed faster when preceded by two-repeated-level trials (670 ms), compared to either four $(753 \mathrm{~ms})$ or six repeated-level trials (762 ms) (see Fig. 3a). There was no effect of the Number of Repeated-Level Trials in the error data (mean error rates were 7, 7, and $8 \%$ in the two, four, and six repeated-level conditions, respectively), indicating that overall accuracy was high and that the pattern of RTs did not reflect a speed/accuracy trade-off.

\section{Switch Direction and Switch Cue}

The RT main effect of Switch Cue $(F(1,11)=32.1$, Mse $=11883.9, \mathrm{P}<0.01)$ reached significance. External switches (637 ms) were faster than internal switches (815 ms). This pattern was reflected in the error data, in which external switches contained fewer errors (4\%) than internal switches (8\%). Although the

\footnotetext{
${ }^{3}$ Studies of sequential priming (e.g., Kirby, 1972) and internal/ external switch cues (e.g., Posner et al., 1980) have a long, established history, and we had no theoretical rationale with which to now conjoin the two literatures. The key aim was to associate behavioral effects with underlying patterns of activation. Given only $\sim 7$ data points per cell and the use of a stringent random-effects model, it seemed very unlikely that any significant interaction effects between these two variables would emerge in the fMRI data. In consequence, any analysis would be liable to a type II error. Nonetheless, we do not dismiss the existence of such interactions and future studies may wish to address such a possibility.
} 
TABLE 1

Contrasts Associated with Significant Brain Activation, Showing Region, Talairach Coordinates, Z Score, and Level of Statistical Significance

\begin{tabular}{|c|c|c|c|c|c|c|}
\hline \multirow[b]{2}{*}{ Contrast } & \multirow[b]{2}{*}{ Region (BA) } & \multirow[b]{2}{*}{ Side } & \multicolumn{3}{|c|}{ Coordinates (mm) } & \multirow[b]{2}{*}{ Z score } \\
\hline & & & $x$ & $\mathrm{y}$ & z & \\
\hline $\begin{array}{l}\text { Two repeated-level trials versus six } \\
\text { repeated level-trials }\end{array}$ & Bilateral medial parietal cortex (7) & $L / R$ & 10 & -38 & 42 & $5.0^{* *}$ \\
\hline \multirow[t]{4}{*}{$\begin{array}{l}\text { Six repeated-level trials versus two } \\
\text { repeated-level trials }\end{array}$} & Motor cortex (4) & $\begin{array}{l}L \\
R\end{array}$ & $\begin{array}{r}-42 \\
34\end{array}$ & $\begin{array}{l}-6 \\
-4\end{array}$ & $\begin{array}{l}54 \\
62\end{array}$ & $\begin{array}{l}5.8^{* *} \\
4.1^{*}\end{array}$ \\
\hline & Premotor cortex (6) & L & -52 & 2 & 38 & $4.9 * *$ \\
\hline & Posterior parietal cortex (7) & L & -30 & -60 & 54 & $4.2^{*}$ \\
\hline & & $\mathrm{R}$ & 34 & -56 & 50 & $4.4^{* *}$ \\
\hline \multirow[t]{2}{*}{ Internal versus external } & Putamen & L & -14 & 4 & 2 & $3.8^{*}$ \\
\hline & & $\mathrm{R}$ & 20 & 4 & 12 & $4.1^{*}$ \\
\hline $\begin{array}{l}\text { Internal global-local versus internal } \\
\text { local-gobal }\end{array}$ & Premotor cortex (6) & $\mathrm{R}$ & 26 & 4 & 56 & $4.9 * *$ \\
\hline \multirow{7}{*}{ Conjunction of internal and external } & Anterior cingulate cortex (24) & $L / R$ & -2 & 10 & 36 & $5.9 * *$ \\
\hline & Pre-SMA (6) & $\mathrm{L} / \mathrm{R}$ & -4 & 4 & 68 & $5.5^{* *}$ \\
\hline & Medial parietal cortex (7) & $\mathrm{L}$ & -6 & -72 & 48 & $5.6 * *$ \\
\hline & Inferior parietal cortex (40) & L & -40 & -28 & 40 & $5.4^{* *}$ \\
\hline & & $\mathrm{R}$ & 46 & -34 & 56 & $4.8^{* *}$ \\
\hline & Postcentral gyrus (3) & L & -54 & -22 & 38 & $5.4^{* *}$ \\
\hline & Fusiform gyrus (19) & L & -44 & -64 & -8 & $5.8^{* *}$ \\
\hline
\end{tabular}

Note. BA, Brodmann area.

** $\mathrm{P}$ value $<0.05$ corrected for entire brain volume.

$* \mathrm{P}$ value $<0.001$ uncorrected for entire brain volume.

main effect of Switch Direction did not reach significance $(F(1,11)=1.3$, Mse $=2558.5, P=0.29)$, the interaction between Switch Direction and Switch Cue was significant $(\mathrm{F}(1,11)=17.9$, Mse $=2659.4, \mathrm{P}<$ $0.01)$. Local-to-global, internally cued switches generated longer latencies than all other Switch DirectionSwitch Cue combinations (see Fig. 3b). None of the two-way interactions were significant in the error data.

\section{fMRI Data}

\section{Number of Repeated-Level Effects}

Significant regions of activation are summarized in Table 1. Switches performed after two repeated-level trials, compared to either four or six repeated-level trials, significantly activated bilateral medial parietal cortex $(Z=5.0$, Talairach coordinates $x=10, y=-38$, $z=42$ ) (see Fig. 4a). In contrast, switches performed after six repeated-level trials significantly activated the left motor cortex $(Z=5.8, x=-42, y=-6, z=54)$, left premotor cortex $(z=4.9, x=-52, y=2, z=38)$, and right posterior parietal lobe $(Z=4.4, x=34, y=$ $-56, z=50)$. At a less stringent threshold $(P<0.001$, uncorrected for multiple comparisons) we observed similar activation in right motor $(Z=4.1, x=34, y=$ $-4, z=62)$ and left parietal $(z=4.2, x=-30, y=$ $-60, z=54$ ) cortices (see Fig. 4b). The same areas were significantly active for switches performed after four compared to six repeated-level trials.
The modulation of motor cortex for switches following either four or six repeated-level trials mapped to the effector system used in the task (i.e., the hand area). Consequently, we next examined whether the lateralized motor cortex activations could be predicted by the hand that subjects used to press the response buttons (recall that each subject responded with the same hand throughout the experiment). The contrast images from the left- and right-hand respondents were subsequently divided into separate groups and, using two-sample t tests, it was then examined whether the observed lateralized motor activations interacted with responding hand. The data indicated significantly greater activation in right motor cortex $(Z=4.5, x=$ $40, y=-16, z=46$ ) for those who responded with only their left hand and significantly greater activation in left motor cortex $(z=4.3, x=-38, y=-22, z=54)$ for those who responded with only their right hand $(P<$ 0.001 , uncorrected for multiple comparisons). This lateralized pattern of activity was observed in all 12 subjects.

\section{Internal/External Switches}

Given our a priori prediction, activation at the uncorrected level ( $P<0.001$, uncorrected for multiple comparisons across the whole brain volume) was accepted when in the lentiform nuclei, ventrolateral thalamus, cerebellum, and right dorsolateral prefrontal 


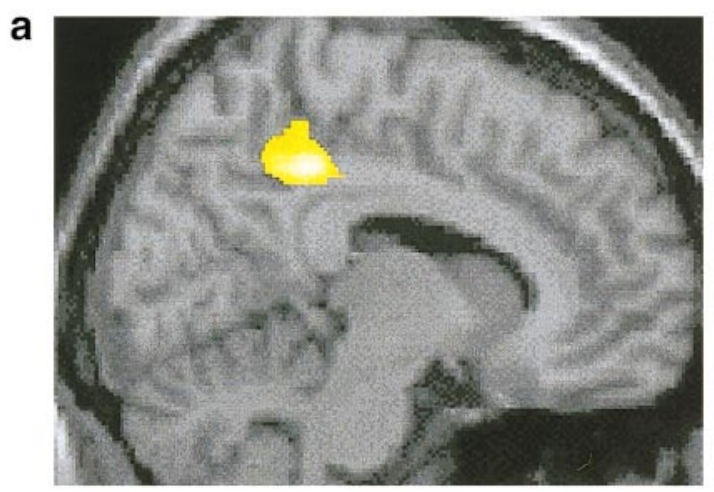

b

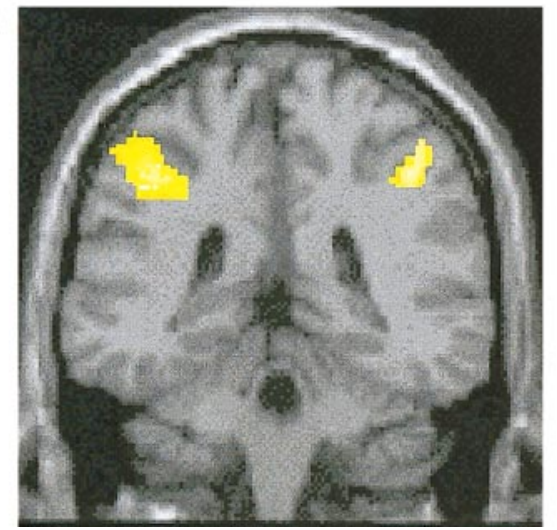

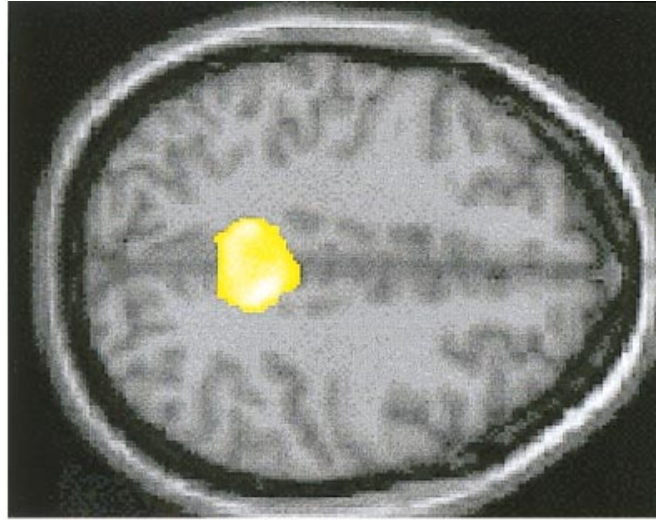

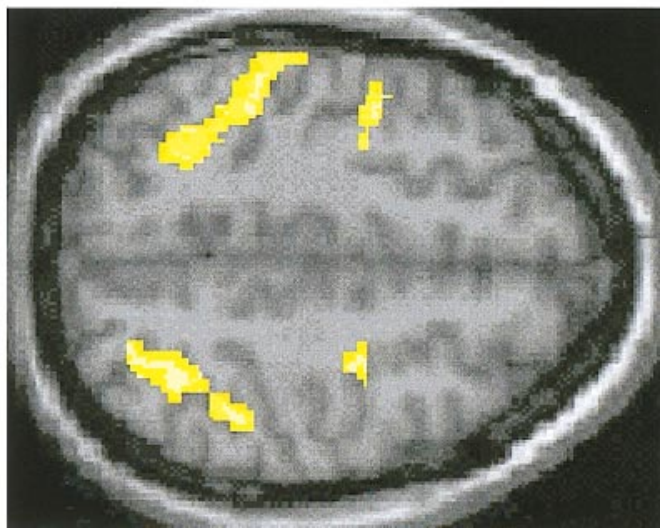

FIG. 4. (a) Activation of the precuneus following switches performed after two repeated-level trials, rendered onto a canonical MRI structural image. (b) Parietal (see coronal section) and parietomotor (see transverse section) activation following switches performed after six repeated-level trials.

cortex. Consistent with this prediction, internal switches significantly activated both the left putamen $(\mathrm{Z}=3.8, x=-14, y=4, z=2)$ and the right putamen $(Z=4.1, x=20, y=4, z=12)$ (see Fig. 5a). Relative to internal switches, external switches were not associated with a distinct pattern of activation.

We then examined whether internal and external switches activated a conjoint, large-scale neural network, as suggested by Rosen et al. (1999). Using the no-switch trials as the baseline, a conjunction analysis was performed across the internal and external conditions. This enabled identification of those significant evoked responses that were common to both conditions (see Price and Friston, 1997, and Friston et al., 1999, for a fuller description of the statistical methodology for conjunction analysis). Activation was observed along the midline, in the anterior cingulate $(Z=5.9, x$ $=-2, y=10, z=36)$, pre-SMA $(Z=5.5, x=-4, y=$ $4, z=68)$, and left precuneus $(z=5.6, x=-6, y=$ $-72, z=48$ ) (see Fig. 5b). Activations were also observed in both left inferior parietal $(Z=5.4, x=-40$, $y=-28, z=40)$ and right inferior parietal cortices ( $z$ $=4.8, x=46, y=-34, z=56$ ) (see Fig. 5c). The left parietal activation extended anteriorly to the left postcentral gyrus $(z=5.4, x=-54, y=-22, z=38)$. We also observed activity around the left fusiform gyrus ( $Z$ =5. $8, x=-44, y=-64, z=-8$ ).

\section{Direction of Switch}

The direction of switch (local to global vs global to local) was not associated with any distinct pattern of activation. However, there was a significant interaction between Switch Cue and Switch Direction. Relative to internal global-to-local switches, internal localto-global switches significantly activated right premotor cortex $(z=4.9, x=26, y=4, z=56)$. There were no significant interactions involving externally cued switches and the direction of switch.

\section{DISCUSSION}

The behavioral data produced two main findings, each of which was mirrored at the level of neural activation. RT costs on changed-level trials were modulated by the number of preceding repeated-level trials; switch trials were longer when performed after four compared to two repeated-level trials, but beyond four trials there was no significant effect on response time (within the range tested). These data confirm that ef- 
a

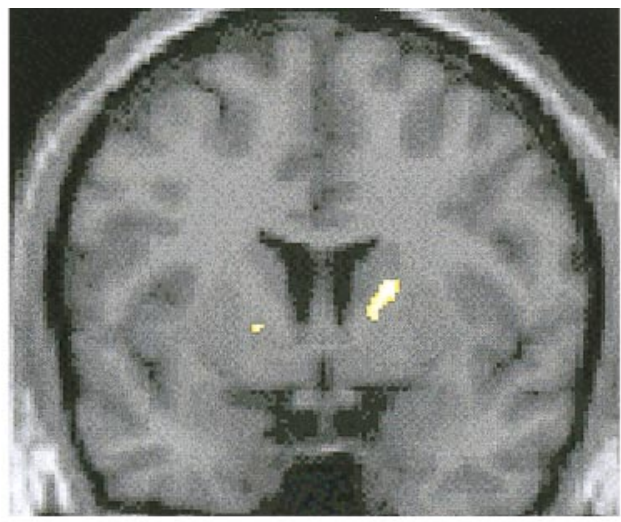

b

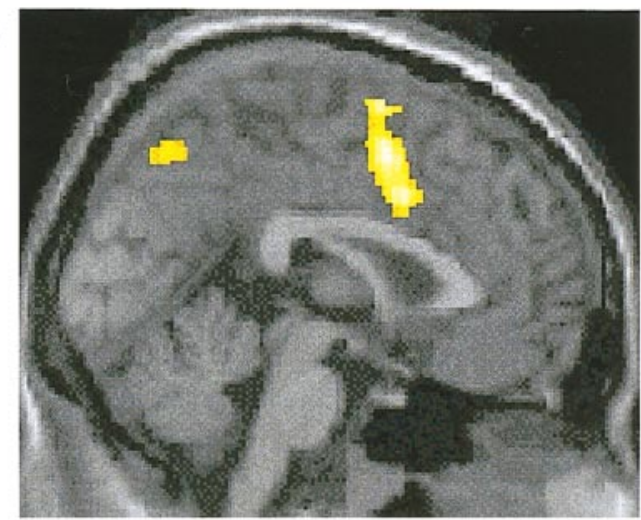

C

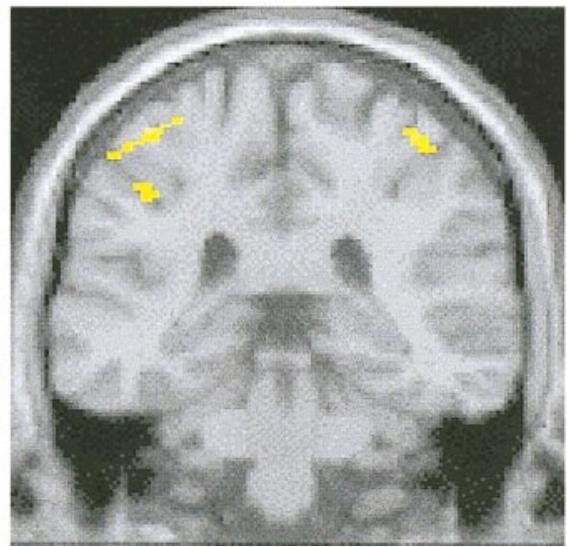

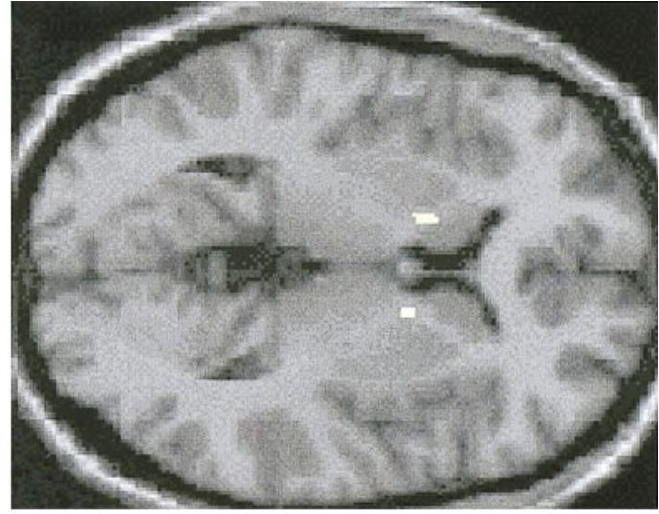
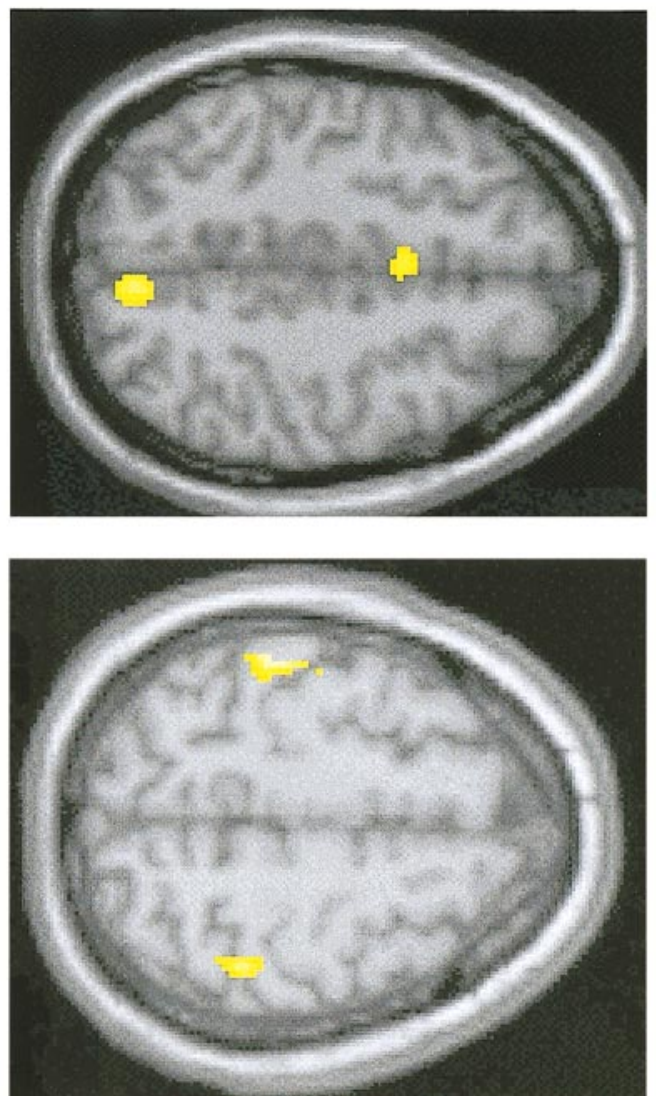

FIG. 5. (a) Activation of left and right putamen in the internal switch condition, rendered onto a canonical MRI structural image. (b) Conjoint activation of cortical midline structures for both internal and external switches. (c) Conjoint activation of bilateral parietal regions for external and internal switches.

fects of target-level information persist beyond the previous trial, even when judgments about target level are not explicitly required. Concordant with the effects reported in spatial cuing tasks, the time taken to identify targets on changed-level trials was faster when the switch was externally rather than internally cued. Previous studies have reported that targets appearing at the level of precedence can be identified faster than targets appearing at the other hierarchical level (Martin, 1979; Navon, 1977). However, by not taking into account both target and precedence level on the previ- ous trial, no-switch trials (in which target and precedence reappear at the same level) could not be disentangled from external switches (in which both target and precedence level switch between trials). In this sense, previous studies were unable to compare external with internal switches. Although our experimental design does not allow us to contrast priming with conscious expectation, we note that automatic priming (which is difficult to extinguish even in severe amnesic and dementing conditions) must account for a substantial proportion of the above effects. 
The coding of target-level information has previously been attributed to left inferior parietal cortex (Robertson et al., 1988). Our data support this claim in that left inferior parietal activation was observed for switches occurring in the four and six repeated-level conditions. However, activation was also observed in right inferior parietal cortex. This is not inconsistent with the Robertson et al. study (1988), since they were unable to test patients with damage in these areas. Our result indicates that the left inferior parietal cortex is not uniquely involved in coding previous target level information.

One further question that arises is why inferior parietal activation was observed for switches following either four or six repeated-level trials but not for two. According to the Attentional Print Model (Robertson, 1996, 1999) target-level information from the previous trial is retained in the form of weights assigned to different spatial-frequency channels. High- and lowspatial-frequency channels resolve local and global targets, respectively, and whichever channel last resolved the target receives a greater weighting in the processing of the next stimulus, leading to level-specific priming. Our data suggest that increases in the number of repeated-level trials interact with the degree of levelspecific priming. As the number of repeated-level trials increases, the weights assigned to each processing stream are modified; the relative assignment of weights to the favored processing stream is much greater after either four or six repeated-level trials, compared to after only two repeated-level trials. After four and six repeated-level trials, the system is therefore relatively more primed, leading to RT savings when the target appears at this level (see Lamb et al., 1988, experiment 2). However, on a changed-level trial this extra priming is disadvantageous, as the input is filtered through the "wrong" spatial-frequency channel. Under these conditions, the operation of the primed channel must in some way be inhibited or overcome if the target is to be promptly resolved. Our parietal activation may have reflected this inhibition, whereby inappropriately primed level-specific processes were deselected. The inhibition could reflect the operation of an automatic process that redistributed weights across spatial-frequency channels or a higher executive mechanism that attentionally reallocated resources. In the latter case, parietal activation may have reflected a breach of expectation, since after only two repeated-level trials, subjects had not yet developed strong expectations of target level, whereas after four they may have done so. This account is consistent with the model proposed by Robertson et al . (1998) and Rafal and Robertson (1995) in which the absence of level-specific priming in parietal patients reflects a failure to either generate or act on expectations of target level.
We note, however, that some studies have failed to demonstrate a close link between spatial frequency and level-specific priming. For example, Lamb and Yund $(1993,1996)$ selectively eliminated the low-spatial-frequency content of their Navon stimuli using contrast balancing, but still observed a level-repetition effect. Similarly, although the posterior parietal lobe has been strongly implicated in shifting attention between hierarchical levels, there remains no direct evidence that it actually utilizes spatial frequency information. For these reasons, a less controversial account of the data may be to focus on the more established motor role of the parietal lobe. Patients with damage to the inferior parietal region sometimes encounter difficulties in initiating movement toward contralesional targets, despite the absence of hemiparesis or sensory loss (Barbieri and De Renzi, 1989), and electrophysiological studies have reported neurons which seem to compute the extrinsic spatial relations between a target and the body, transforming perceptual information into a pattern of proximal movements required for reaching (Andersen et al., 1997). Studies of this nature have led to suggestions that the region may be better viewed as a sensorimotor interface than as either strictly perceptual or strictly motor (Mattingley et al., 1998).

In the present context, it may be that the inferior parietal activation reflected the inhibition of an inappropriately planned response. As the number of repeated-level trials increases, the association between target level and the execution of a motor response increases; i.e., a motor response becomes conditional on first coding the hierarchical level at which the target last appeared. On a repeated-level trial this priming will lead to RT savings. However, on a changed-level trial this response preparation is inappropriate since a switch must be performed before the target can be identified. In this sense, the activation may reflect inhibitory effects at the level of response preparation, rather than sensory disambiguation (i.e., filtering the input at the correct spatial frequency).

Switches performed after four and six repeated-level trials also activated motor and premotor cortex. This motor/premotor activation was not only contralateral to the hand used to perform the response, but also mapped specifically to the motor hand area. The means by which global and local perceptual processing modulates motor output is poorly understood, but we are able to discount several trivial interpretations of the activation. If motor activity increased as a function of button presses (over the range tested) then differences would be expected between four and six, as well as between four and two and six and two. The data do not support this prediction. Further, the RT data indicate a difference between the two and the six condition, and it seems unlikely that this would have stemmed solely from having to make a few extra button presses before 
performing a switch. Previous studies have also demonstrated that activity in motor cortex can be moderated by increases in the frequency of button presses (e.g., J äncke et al., 1998; Rao et al., 1996). However, in the present study, buttons were always pressed with the same frequency (approximately every $3 \mathrm{~s}$ ) and so this explanation can be discounted.

In our study, the important point to note is that a button press was made on every trial, and so the motor activation should not reflect motor execution (although in the absence of EMG recording, one cannot be absolutely certain of this). We accordingly suggest that the activation may reflect a form of inhibitory activity. Such a claim is consistent with M1 activation reported in a recent priming experiment (Dehaene et al., 1998). There subjects were asked to use one hand to press one response key if a visually presented number was greater than 5 and the other hand if it was less. A masked prime appeared prior to target presentation, which was either congruent or incongruent with the target. On correctly primed trials, early covert activity was observed on the correct response side. On incorrectly primed trials, covert induced motor activity on the incorrect response side preceded overt motor-related activity on the correct response side. The authors reasoned that this incongruent activity reflected a response bias that had to be overcome before an appropriate response could be made and that it demonstrated a causal relationship between perceptual priming and motor activity. Likewise, in the present study it is possible that one downstream effect of perceptual priming was to prepare motor cortex for an ensuing response. Activation in the high prime condition may have reflected a deprogramming of this motor readiness while unprimed level-specific processes were activated and the correct target level coded. At the very least, it seems plausible that the longer RTs are, in part, due to a longer preparatory response in the motor areas.

Switches after two repeated-level trials, compared to either four or six repeated-level trials, activated the precuneus. This result provides further evidence for the differential effects of the number of repeated-level trials on hierarchical switching. Activation of this midline structure has been reported in other local/global experiments in which subjects must either sustain attention to a hierarchical level (Heinze et al., 1998) or switch between hierarchical levels (Fink et al., 1997). In the Heinze et al. (1998) study, precuneus activation was reported in a sustained attention task in which subjects had to identify the target at one level throughout the experiment. In contrast, in the Fink et al. (1997) study, this activation was absent for the sustained attention condition, but present in a switching condition in which target level changed at frequent, random intervals across the scanning period. The results of the present study support the claim that this structure is involved in hierarchical switching, although we were unable to test whether the precuneus was also active in the sustained attention condition. We note, however, that the measure of sustained attention in the current study differed from that in the Fink et al. (1997) study; in their study subjects were instructed to attend to only one hierarchical level across a particular block of trials, whereas in the current study target level was unknown before each trial. Fink et al. (1997) suggested that the precuneus may index the greater attentional demands of increased switching rate. This is consistent with the greater precuneus activation seen here after two compared to six repeated-level trials, since in the former condition, both the number of trials and the length of time from the last switch would have been smaller.

One further issue addressed in the current study was how, in neural terms, those mechanisms that code internal and external cues modulate local/global processing on changed-level trials. Internal switches, relative to external switches, activated right and left putamen. This structure constitutes part of a loop between other basal ganglia/thalamic nuclei and the prefrontal cortex (Alexander and Crutcher, 1990) and has been implicated in several other kinds of internally mediated, attentional switching task. For example, Rosen et al. (1999) observed similar activity following covert shifts of attention away from invalid, peripheral targets. Brown and Marsden (1988) reported impaired Stroop performance in Parkinson's patients, who suffer from striatal (i.e., putamen) degeneration. Notably, patients performed most poorly when the nature of the response was determined by the patients themselves, i.e., cues indicating the dimension that they must report were withdrawn. In contrast, in our study external switches, relative to internal switches, did not reveal any significant activation. One reason for this may be that stimulus precedence always induced an external component into internally cued switches. If internal switches contained the same cognitive components as external switches, plus an additional switch needed to overcome the effects of precedence, then a simple subtraction analysis between external and internal trials would not reveal any significant activation.

Previous studies have also indicated that both internally and externally cued attentional shifts draw on a closely overlapping system, including bilateral premotor cortex, anterior cingulate, pre-supplementary motor area, frontal eye fields, and posterior parietal cortex (e.g., N. Kim et al., 1999; Nobre et al., 1997; Rosen et al., 1999). Our data are consistent with these observations, as activation was observed in pre-SMA, bilateral parietal, and anterior cingulate cortices, as well as the fusiform gyrus. It appears that for both local/gl obal and spatial cuing tasks, internal and external switching is mediated by a similar pattern of neural activity. Spatial cuing tasks typically use targets defined by a pe- 
ripheral box that changes in either luminance or color (Nobre et al., 1997; Rosen et al., 1999) or that briefly contains a cross (N. Kim et al., 1999). In this sense, external cues are induced by a sudden change in stimulus attribute. In the present study, targets appeared centrally within a Navon stimulus, and external cues were induced by intrinsic stimulus precedence, rather than by a sudden change in the display. The overlap in activation between these different display conditions indicates that common mechanisms underlie switching between spatial scales and between lateralized locations and that these mechanisms are not specific to the nature of the external cue. Although strongly suggestive, we note that these similarities do not necessarily imply that both kinds of switches are underlain by the same cerebral architecture.

Corbetta et al. (2000) recently demonstrated in a spatial cuing paradigm that target detection preferentially engages the temporal-parietal junction, inferior parietal, and precuneus, while the reorientation of attention following a cue that appears before target presentation preferentially engages the intraparietal sulcus. This sensitivity of the inferior parietal lobe to visual cues rather than targets was also reported in another recent spatial cuing experiment (Hopfinger et al., 2000). Regrettably, the precedence cues used in local/global experiments cannot be easily disentangled from target effects. This is because both cue and target must appear simultaneously, as they constitute different aspects of the same stimulus (cue = precedence, target = letter). Nonetheless, in support of Corbetta et al. (2000) we observed large activation across posterior parietal cortex in the switch versus no-switch contrast (the conjunction analysis). This is consistent with their claim that both effects are traceable to posterior parietal lobe.

One question that arises is why this pattern of performance for external/internal switches is found inconsistently in the behavioral literature; Robertson (1996, experiment 1) observed the effect, while Lamb and Yund (1996) did not. Other studies have demonstrated a symmetrical pattern of costs and benefits when a target is more likely to occur at a given level or if subjects are informed that one level is more important than the other. Thus, although stimulus precedence may initially facilitate processing at one level, it is likely that other biases, such as expectation, can counteract these effects. The likelihood of observing external effects may therefore be enhanced by, as in the present study, constructing stimuli with stronger precedence. The strength of local or global precedence in previous studies is often difficult to discern, but our hypothesis leads us to infer that these were either weak and/or overcome by other factors, since switches away from precedence were not reliably slower than switches toward precedence. As demonstrated by Martin (1979) global precedence is not a predefined visual property, and it seems that in many previous studies experimenters were more concerned with examining the role of expectation on switching performance rather than optimizing certain stimulus attributes such as precedence.

In conclusion, we report that both temporal context (i.e., how many trials ago a change was last made) and switch cue (as determined by stimulus precedence) affect the ease with which local/global targets can be identified in changed-level trials. We further identify the neural correlates associated with these changes in switching efficiency; changed-level trials that are preceded by only two repeated-level trials preferentially activate the precuneus while those preceded by either four or six repeated-level trials activate a parietalmotor network. I nternally mediated switches activate right and left putamen, while both internal and external switches conjointly activate a large-scale bilateral frontal-parietal network. These data indicate that the switch costs observed in Navon stimuli are modulated by a variety of distinct structures, each of which is sensitive to different aspects of the switch. Furthermore, the findings illustrate the possibility of inferring underlying anatomical dissociations from RT data.

\section{ACKNOWLEDGMENTS}

We thank R. E. Passingham for neuroanatomical advice. D.T.W., P.W.H., and J .C.M. are supported by the Medical Research Council. C.B. and R.J .D. are supported by the Wellcome Trust.

\section{REFERENCES}

Alexander, G. E., and Crutcher, M. D. 1990. Preparation for movement: Neural representations of intended direction in three motor areas of the monkey. J . Neurophysiol. 64: 133-150.

Boles, D. B., and Karner, T. A. 1996. Hemispheric differences in global versus local processing: Still unclear. Brain Cognit. 30: 232-243.

Briggs, G. G., and Nebes, R. D. 1975. Patterns of hand preference in a student population. Cortex 11: 230-238.

Brown, R. G., and Marsden, C. D. 1988. Internal versus external cues and the control of attention in Parkinson's disease. Brain 111: 323-345.

Corbetta, M., Kincade, J. M., Ollinger, J . M., McAvoy, M. P., and Shulman, G. L. 2000. Voluntary orienting is dissociated from target detection in human posterior parietal cortex. Nat. Neurosci. 3: 292-296.

Dehaene, S., Naccache, L., Le Clerc, H. G., Koechlin, E., Mueller, M., Dehaene-Lambertz, G., van der Moortele, P. F., and Le Bihan, D. 1998. Imaging unconscious semantic priming. Nature 395: 597600.

Delis, D. C., Robertson, L. C., and Efron, R. 1986. Hemispheric specialization of memory for visual hierarchical stimuli. Neuropsychologia 24: 205-214.

Fink, G. R., Halligan, P. W., Marshall, J . C., Frith, C. D., Frackowiak, R. S., and Dolan, R. J . 1997. Neural mechanisms involved in the processing of global and local aspects of hierarchically organized visual stimuli. Brain 120: 1779-1791. 
Fink, G. R., Marshall, J . C., Halligan, P. W., Frith, C. D., Frackowiak, R. S., and Dolan, R. J . 1998. Hemispheric specialization for global and local processing: The effect of stimulus category. Proc. R. Soc. London B 264: 487- 494.

Friston, K. J ., Holmes, A. P., Price, C. J ., Büchel, C., and Worsley, K. J. 1999. Multisubject $\mathrm{fMRI}$ studies and conjunction analyses. Neurol mage 10: 385-396.

Halligan, P. W., and Marshall, J . C. 1994. Toward a principled explanation of unilateral neglect. Cognit. Neuropsychol. 11: 167-206.

Heinze, H. J ., Hinrichs, H., Scholz, M., Burchert, W., and Mangun, G. R. 1998. Neural mechanisms of global and local processing: A combined PET and ERP study. J . Cognit. Neurosci. 10: 485- 498.

Hopfinger, J. B., Buonocore, M. H., and Mangun, G. R. 2000. The neural mechanisms of top- down attentional control. Nat. Neurosci. 3: 284-291.

J änke, L., Specht, K., Mirzazade, S., Loose, R., Himmelbach, M., Lutz, K., and Shah, J. 1998. A parametric analysis of the 'rate effect' in the sensorimotor cortex: A functional magnetic resonance imaging analysis in human subjects. Neurosci. Lett. 252: 37- 40.

Kim, N., I vry, R., and Robertson, L. C. 1999. Sequential priming in hierarchically organized figures: Effects of target level and target resolution. J . Exp. Psychol. Hum. Percept. Perform. 25: 715-729.

Kim, Y. H., Gitelman, D. R., Nobre, A. C., Parrish, T. B., LaBar, K. S., and Mesulam, M. M. 1999. The large-scale neural network for spatial attention displays multifunctional overlap but differential asymmetry. Neurol mage 9: 269-277.

Kirby, N. H. 1972. Sequential effects in serial reaction time. J . Exp. Psychol. 96: 32-36.

Lamb, M. R., and Robertson, L. C. 1988. The processing of hierarchical stimuli: Effects of retinal locus, locational uncertainty, and stimulus identity. Percept. Psychophys. 44: 172-181.

Lamb, M. R., and Yund, E. W. 1993. The role of spatial frequency in the processing of hierarchically organized stimuli. Percept. Psychophys. 54: 773-784.

Lamb, M. R., and Yund, E. W. 1996. Spatial frequency and attention: Effects of level-, target-, and location-repetition on the processing of global and local forms. Percept. Psychophys. 58: 363-373.

Lamb, M. R., London, B., Pond, H. M., and Whitt, K. A. 1998. Automatic and controlled processes in the analysis of hierarchical structure. Psychol. Sci. 9: 14-19.

Lamb, M. R., Yund, E. W., and Pond, H. M. 1999. Is attentional selection to different levels of hierarchical structure based on spatial frequency? J . Exp. Psychol. Gen. 128: 88-94.

Martin, M. 1979. Local and global processing: The role of sparsity. Memory Cognit. 7: 476- 484.
Navon, D. 1977. Forest before trees: The precedence of global features in visual perception. Cognit. Psychol. 9: 353-383.

Nobre, A. C., Sebestyen, G. N., Gitelman, D. R., Mesulam, M. M, Frackiowiak, R. S., and Frith, C. D. 1997. Functional localization of the system for visuo-spatial attention using positron emission tomography. Brain 120: 515-333.

Palmer, S. E. 1977. Hierarchical structure in perceptual representation. Cognit. Psychol. 9: 441- 474.

Posner, M. I., Synder, C. R. R., and Davidson, B. J . 1980. Attention and the detection of signals. J . Exp. Psychol. Gen. 109: 160-174.

Posner, M. I., Walker, J. A., Friedrich, F. A., and Rafal, R. 1984. Effects of parietal injury on converting orienting of attention. J . Neurosci. 4: 1863-1874.

Price, C. J ., and Friston, K. J . 1997. Cognitive conjunction: A new approach to brain activation experiments. Neurol mage 5: 261270.

Rafal, R., and Robertson, L. C. 1995. The neurology of visual attention. In Handbook of Cognitive Neuroscience (M. Gazzaniga, Ed.). MIT Press, Cambridge, MA.

Rao, S. M., Bandettini, P. A., Binder, J . R., Bobholz, J . A., Hammeke, T. A., Stein, E. A., and Hyde, J. S. 1996. Relationship between finger movement rate and functional magnetic resonance signal change in human primary motor cortex. J. Cereb. Blood Flow Metab. 16: 1250-1254.

Robertson, L. C. 1996. Attentional persistence for features of hierarchical patterns. J . Exp. Psychol. Gen. 125: 227-249.

Robertson, L. C. 1999. Spatial frequencies as a medium for guiding attention: Comment on Lamb, Yund and Pond, (1999). J . Exp. Psychol. Gen. 128: 95-98.

Robertson, L. C., Lamb, M. C., and Knight, R. T. 1988. Effects of lesions of temporal-parietal junction on perceptual and attentional processing in humans. J . Neurosci. 8: 3757-3769.

Robertson, L. C., Egly, R., Lamb, M. R., and Kerth, L. 1993. Spatial attention and cuing to global and local levels of hierarchical structure. J . Exp. Psychol. Hum. Percept. Perform. 19: 471- 487.

Rosen, A. C., Rao, S. M., Caffarra, P., Scaglioni, A., Bobholz, J . A., Woodley, S. J ., Hammeke, T. A., Cunningham, J . M., Prieto, T. E., and Binder, J. R. 1999. Neural basis of internal and external spatial orienting: A functional MRI study. J . Cognit. Neurosci. 11: 135- 152.

Ward, L. M. 1982. Determinants of attention to local and global features of visual forms. J . Exp. Psychol. Hum. Percept. Perform. 8: 562-581. 